\title{
Doble fijación para la rotura de bíceps distal: Descripción de la técnica y evaluación de resultados
}

\section{Double Fixation for Distal Biceps Rupture: Technique Description and Results Evaluation}

\author{
Miguel A. Capomassi ${ }^{1} \quad$ Manuel A. Vélez $^{1} \quad$ R. Martín Gardenal ${ }^{1}$ \\ ${ }^{1}$ Instituto de Traumatología Dr. Jaime Slullitel, Rosario, Santa Fe, \\ Argentina \\ Address for correspondence Miguel A. Capomassi, MD, Instituto de \\ Traumatología Dr. Jaime Slullitel, San Luis 2534, CP: 2000, Rosario, \\ Santa Fe, Argentina (e-mail: miguelcapomassi@gmail.com).
}

Rev Iberam Cir Mano 2017;45:16-23.

\begin{abstract}
Resumen
Introducción La ruptura del bíceps distal es una lesión infrecuente producida por la aplicación de una carga excéntrica sobre el codo flexionado. El objetivo de este trabajo es analizar nuestros resultados para la reinserción del bíceps distal mediante la técnica de doble fijación con botón de anclaje cortical y tornillo interferencial de biotenodesis a través de una incisión anterior única, con una casuística mayor a la presentada con anterioridad. Material y Método Entre agosto de 2008 y agosto de 2015, se intervino 24 casos de rotura del bíceps distal tratados quirúrgicamente por vía anterior limitada. Los 22 últimos casos consecutivos fueron tratados con doble fijación utilizando botón extracortical y tornillo de biotenodesis, y son el grupo de estudio de este artículo. Todos eran hombres, con una mediana de edad de 39,5 años (rango 22-59). Los pacientes fueron evaluados con el Cuestionario DASH y la valoración Subjetiva/Objetiva de Andrews y Carson, la fuerza de flexión y supinación se controló con la escala del British Medical Research Council modificada por Mackinnon y Dellon (M0-M5).

Resultados El valor promedio del cuestionario DASH fue de $1,7(0-31,6)$ y el de Andrews y Carson, de 197,5 (135-200), con 20 resultados excelentes, 1 bueno y 1 regular. Todos consiguieron una fuerza M5 para la flexión y la supinación de antebrazo.

Palabras Clave

- tendón del bíceps

- rotura distal

- técnica de doble fijación
Abstract
Keywords
- biceps tendón
- distal ruptura
- double fixation technique

El tiempo promedio de seguimiento fue de 17,5 meses (10-38). En tres pacientes se registraron complicaciones, todos con una recuperación total.

Conclusión La técnica descrita demostró ser segura y eficaz, con resultados satisfactorios y baja tasa de complicaciones.

Nivel de Evidencia: IV. Serie de Casos.

Purpose Distal biceps rupture is an unusual lesion produced after an eccentric load is applied to the bent elbow. The aim of this study is to analyze our results for the reinsertion of the distal biceps with a double fixation technique of cortical button and biotenodesis interference screw through a single anterior incision, with a larger number of cases cases than previously submitted.
\end{abstract}

received

October 25, 2015

accepted

March 25, 2017
DOI https://doi.org/

10.1055/s-0037-1602793.

ISSN 1698-8396.
Copyright $\odot 2017$ by Thieme Revinter

Publicações Ltda, Rio de Janeiro, Brazil
License terms

(c) $(1) \$$ 
Methods Between August 2008 and August 2015, 24 cases of distal biceps rupture, surgically treated through an anterior limited procedure. The last 22 consecutive cases were treated with double fixation using an extracortical button and biotenodesis screw and are the group tudied in this paper. All patients were males with a mean age of 39,5 years (range 22-59). Patients were evaluated using the DASH questionnaire and the subjective/ objective scoring system of Andrews and Carson, flexion and supination strengths were assessed with the British Medical Research Council scale, modified by Mackinnon and Dellon (M0-M5).

Results The mean DASH score was 1,7 (0-31,6), and the mean Andrews and Carson score was 197,5 (135-200), with 20 excellent, 1 good and 1 regular results. All patients achieved a M5 (level of) flexion and supination strenght of the forearm. The median follow-up period was 17,5 months (10-38). Complications were registered in three patients that recovered totally.

Conclusion The described technique proved to be both reliable and effective, giving satisfactory results with a low complication rate.

Level of Evidence IV. Case Series.

\section{Introducción}

La rotura del tendón distal del bíceps es una lesión de baja incidencia producida por la aplicación de una fuerza excéntrica sobre el codo flexionado. Sin embargo, en los últimos años, se ha observado un franco incremento como resultado de las excesivas demandas aplicadas al miembro superior, particularmente laborales y deportivas, así como también por la expansión de las actividades físicas a la población de edad promedio.

La bibliografía demuestra que los resultados funcionales obtenidos con la reparación quirúrgica de esas lesiones son claramente superiores a los del tratamiento conservador ${ }^{1,2}$ $\mathrm{y}$ es de primera elección en pacientes activos con alta demanda funcional. Se prefieren las técnicas de anclaje anatómico del tendón a las reparaciones funcionales o indirectas mediante simple sutura al braquial anterior. El tratamiento conservador se reserva para pacientes añosos con actividades que toleran una variable pérdida de la fuerza de flexión del codo y de potencia supinadora del antebrazo. En la actualidad, se utilizan para la reconstrucción anatómica una gama de recursos que incluyen suturas tipo pull-out, tornillos interferenciales, botones corticales y arpones. $^{3-12}$ Los abordajes han variado a lo largo del tiempo. Desde la original vía anterior extendida de Henry ${ }^{13}$ y el doble abordaje propuesto por Boyd y Anderson. ${ }^{14}$ En la actualidad y propiciado por los nuevos implantes disponibles, los accesos quirúrgicos tienden a reducirse, e incluso realizarse bajo asistencia artroscópica, ${ }^{15}$ reduciendo el trauma quirúrgico y las potenciales complicaciones.

El objetivo de este trabajo es analizar los resultados funcionales obtenidos en un grupo de pacientes tratados, de forma consecutiva, con botón de anclaje cortical y tornillo interferencial de biotenodesis a través de una incisión anterior única.

\section{Material y Método}

Se trata de un estudio observacional descriptivo de los pacientes con rotura de bíceps distal tratados por vía anterior limitada en el período comprendido entre agosto de 2008 y agosto de 2015. Los dos primeros casos, se resolvieron mediante fijación única con tornillo interferencial, por lo que se excluyeron de este estudio. Los 22 casos consecutivos restantes fueron tratados con doble fijación utilizando botón extracortical y tornillo de biotenodesis. Ésta es una serie ampliada que incluye nuestros casos ya presentados de forma preliminar en el año 2013. ${ }^{16}$

Todos los pacientes eran hombres, con un promedio de edad de 39,5 años (rango de 22 a 59). La serie incluye 12 trabajadores de fuerza, 9 deportistas ( 5 jugadores de rugby, 1 tenista, 2 levantadores de pesas, 1 boxeador) y 1 cirujano general con práctica deportiva recreativa. En 13 casos (59,1\%), el miembro afectado era el dominante. La reconstrucción se realizó dentro de las primeras 4 semanas (período agudo) en 18 pacientes, en tanto que los 4 restantes fueron operados más tarde del mes (período crónico). La mediana del tiempo entre el traumatismo y la cirugía fue de 17,5 días (rango de 5 a 95), demora motivada en algunos casos por consulta tardía o retraso en la obtención de los implantes.

La técnica quirúrgica empleada fue la siguiente. El paciente se coloca en decúbito supino con el hombro en abducción de 90 grados y codo extendido sobre la mesa de miembro superior. La intervención se realiza bajo anestesia regional o general. Se utiliza manguito hemostático a $270 \mathrm{~mm} \mathrm{Hg}$ para una mejor visualización de las estructuras durante el abordaje, aunque no es indispensable. Se realiza una incisión longitudinal de $35-40 \mathrm{~mm}$ en la cara anterior del antebrazo, $30 \mathrm{~mm}$ distal al pliegue del codo. Se identifican y protegen las venas superficiales y, en especial, el 
nervio antebraquial cutáneo lateral en el espesor del tejido celular subcutáneo. Se avanza en profundidad a través del intervalo entre el pronador redondo y el braquiorradial siguiendo el trayecto del tendón avulsionado que conduce directamente a la tuberosidad bicipital. En ese punto, es necesario identificar $\mathrm{y}$, si fuera necesario, ligar o coagular las tributarias transversales del plexo venoso de Henry, cuyo sangrado complica los siguientes pasos de la técnica. Mediante disección digital se localiza el cabo distal del tendón del bíceps hacia el proximal, se libera y regulariza en su extremo aplicando un punto de Krackow con sutura 2/0 tipo FiberLoop ${ }^{\circledR}$ (Arthrex Inc., Naples, FL), procurando que el diámetro del tendón preparado no supere los $7 \mathrm{~mm}$. En máxima supinación, se palpa y expone la tuberosidad bicipital del radio protegiendo la integridad del nervio interóseo posterior (evitando el uso de separadores en el lado radial), y se resecan los restos de inserciones tendinosas. Sobre una aguja guía de $3,2 \mathrm{~mm}$ que atraviesa ambas corticales se comienza el fresado progresivo de la cortical anterior hasta $8 \mathrm{~mm}$. Los dos cabos del punto de Krackow se pasan por el botón cortical de $12 \mathrm{~mm}$ BicepsButton ${ }^{\circledR}$ (Arthrex Inc., Naples, FL) y se introducen a través del túnel óseo saliendo por la segunda cortical. Es aconsejable, en ese punto, comprobar la correcta posición final con intensificador de imágenes. Traccionando los cabos de sutura con el codo en flexión de 60 grados y supinación máxima, el tendón se introduce en el túnel óseo hasta la cortical opuesta. Luego, con técnica habitual de montaje, se introduce el tornillo de biotenodesis de $7 \times 10 \mathrm{~mm}$ tipo PEEK Tenodesis Screw ${ }^{\circledR}$ (Arthrex Inc., Naples, FL) del lado radial del tendón, para lograr un anclaje más anatómico y mayor efecto supinador. Finalmente, se evalúa el rango de movimiento articular y la estabilidad de la fijación. Se realiza un cierre por planos, se coloca un vendaje acolchado y una férula posterior en 90 grados de flexión del codo.

Esa técnica fue modificada ligeramente en los pacientes operados en el período crónico. En 5 de ellos, se necesitó una incisión complementaria en la cara anterior del tercio distal del brazo, con el propósito de liberar adherencias músculotendinosas y lograr una mayor excursión distal del tendón, mientras que, en el Caso 2, se utilizó un injerto de palmar menor cuádruple para llegar a la tuberosidad bicipital (ese caso se operó 60 días desde la lesión y presentaba una marcada retracción por la rotura de la expansión aponeurotica del bíceps). En ninguno de esos casos, se varió la construcción del anclaje distal.

Como protocolo rehabilitador, los pacientes comienzan la rehabilitación a la semana, con ortesis que se puede colocar y retirar. Se realiza un bloqueo progresivo de la extensión procurando la flexión y pronosupinación pasivas completas. Se suspende el uso de la ortesis a las 3 semanas, y se inicia el uso activo del miembro con carga limitada. Al mes, las cargas se aumentan progresivamente con fortalecimiento muscular. A los 4 meses, se permite la carga máxima deportiva o laboral (-Figs. 1-4).

A todos los pacientes, se les evaluó con el Cuestionario DASH $^{17}$ y el Puntaje Subjetivo/Objetivo de Andrews y Carson ${ }^{18}$; se controló la fuerza de flexión y supinación con la escala del
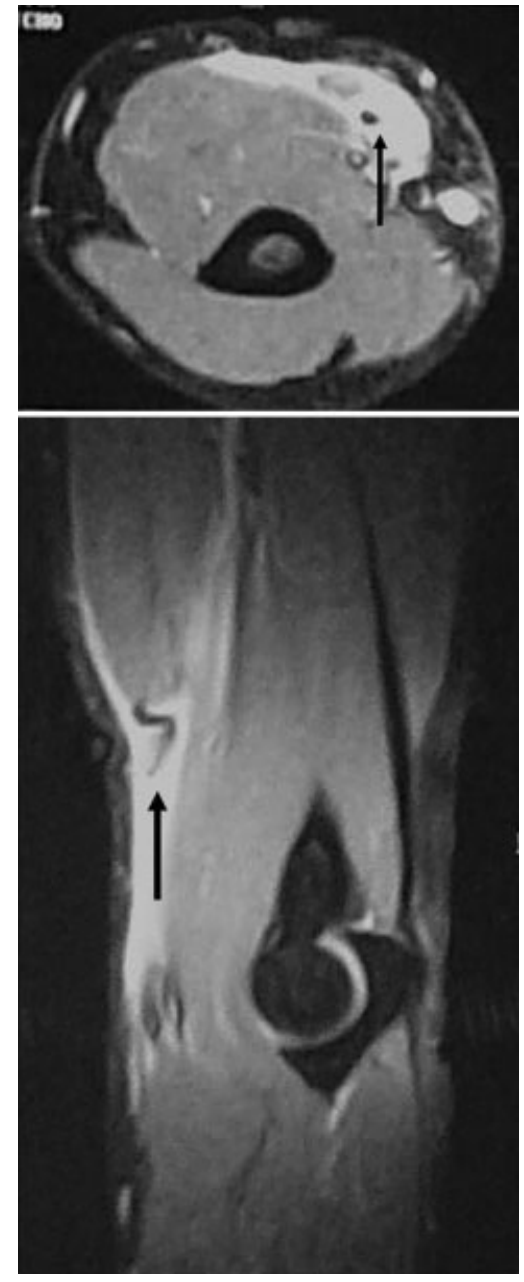

Fig. 1 Imagen del tendón bicipital avulsionado en los planos axial y sagital de la resonancia magnética.

British Medical Research Council ${ }^{19}$ modificada posteriormente por Mackinnon y Dellon ${ }^{20}$ (M0-M5). Los resultados se expresaron como mediana (rango mínimo-máximo).

\section{Resultados}

En el cuestionario DASH se obtuvo una mediana de 1,7 (rango de 0 a 31,6) y el de Andrews y Carson, de 100 (rango 95 a 100) para el componente objetivo, 97,5 (rango de 40 a 100) para el componente subjetivo y 197,5 (rango de 135 a 200) para el total; en 20 pacientes, se logró un resultado excelente, en 1 bueno y en 1 regular. Todos consiguieron una fuerza M5 para flexión y supinación del antebrazo. La mediana del seguimiento fue de 17,5 meses (rango de 10 a 38). Tres pacientes tuvieron complicaciones: una dehiscencia de herida con fístula aséptica y dos neuropraxias radial alta, a nivel del tercio proximal del brazo, motivada por el manguito hemostático, todas con recuperación completa (Tabla $\mathbf{1}$ ).

\section{Discusión}

En la actualidad, la avulsión distal del bíceps representa el $10 \%$ de las roturas bicipitales y es el resultado de una contracción excéntrica del músculo por la abrupta extensión 


\begin{tabular}{|c|c|c|c|c|c|c|c|c|c|c|c|c|c|c|c|c|c|c|c|c|c|}
\hline 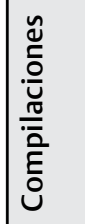 & 운 & 을 & i & 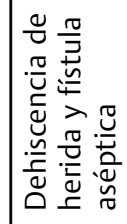 & 2o & 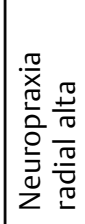 & $\frac{1}{2}$ & 운 & z & z & z & z & z & 2 & 2 & \begin{tabular}{l|l}
0 \\
$z$
\end{tabular} & \begin{tabular}{l|l}
0 \\
$z$
\end{tabular} & \begin{tabular}{l|l}
0 & 0 \\
$z$ &
\end{tabular} & 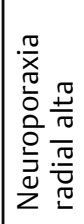 & 울 & i \\
\hline 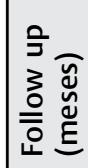 & $\stackrel{\bullet}{N}$ & $\stackrel{\stackrel{े}{m}}{ }$ & $\stackrel{\infty}{m}$ & $\stackrel{\Delta}{\sim}$ & $\approx$ & $\stackrel{n}{\sim}$ & $\tilde{m}$ & $\stackrel{\stackrel{n}{N}}{ }$ & $\stackrel{\infty}{-}$ & $\stackrel{\infty}{-}$ & $\approx$ & $\stackrel{\operatorname{Ln}}{\sim}$ & \pm & $\circ:$ & $\pm \mid ?$ & $\simeq$ & $\circ \circ$ & 인 & $\bar{\sim}$ & $\circ$ & $\cong$ \\
\hline 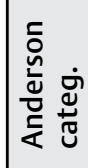 & 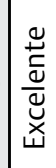 & 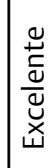 & 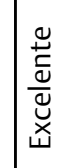 & 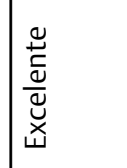 & 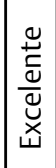 & 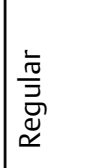 & 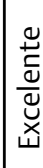 & 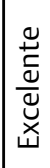 & 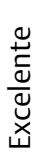 & $\stackrel{\circ}{\frac{0}{0}}$ & 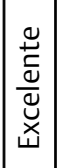 & 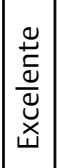 & 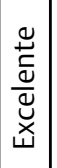 & 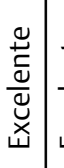 & 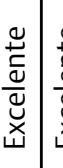 & 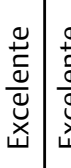 & 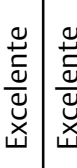 & 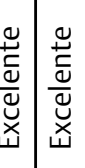 & 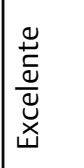 & 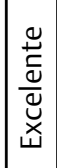 & 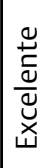 \\
\hline 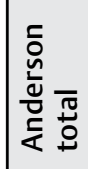 & ํㅡㅁ & $\stackrel{\text { D }}{\text { N }}$ & 으 & Ф & $\stackrel{\llcorner}{\infty}$ & $\stackrel{\stackrel{n}{m}}{\longrightarrow}$ & Цِ & 으 & ঃ & $\stackrel{\stackrel{n}{\Omega}}{=}$ & $\stackrel{\text { 음 }}{\leftarrow}$ & 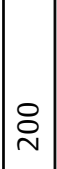 & $\stackrel{\stackrel{\varrho}{\circ}}{\square}$ & 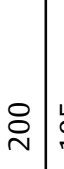 & ڤૂ & $\stackrel{i}{i}$ & 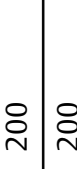 & 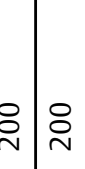 & 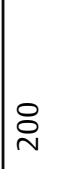 & 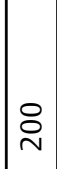 & นn \\
\hline
\end{tabular}

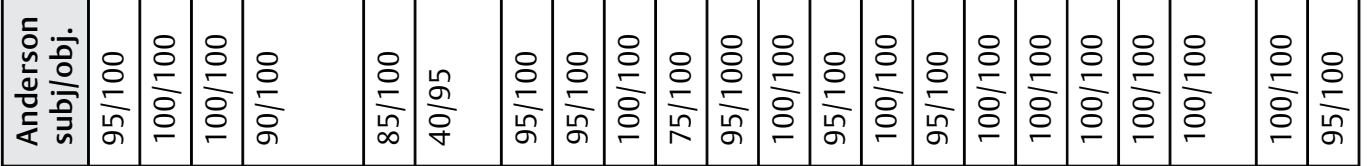

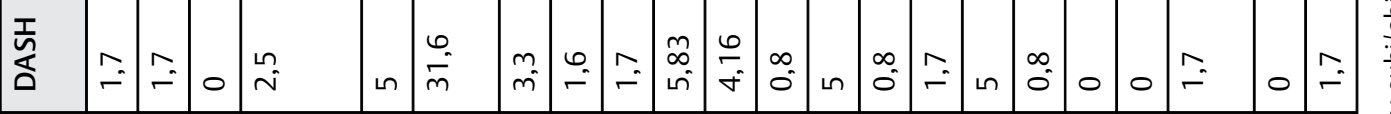

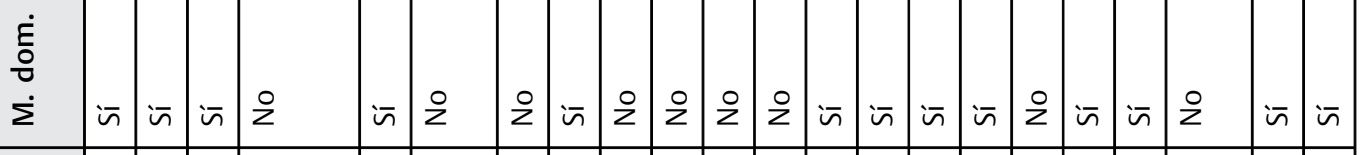

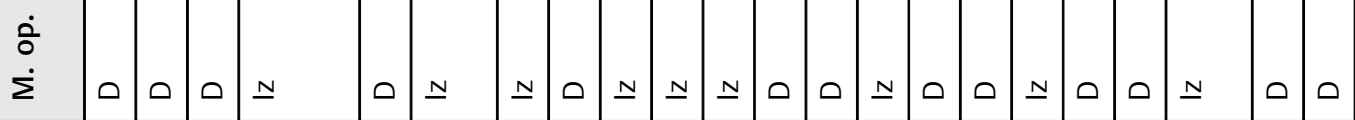

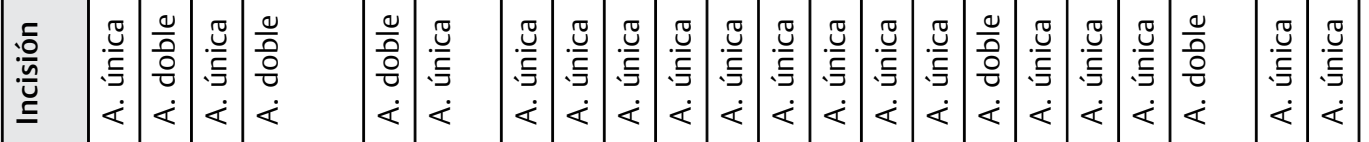
毫药

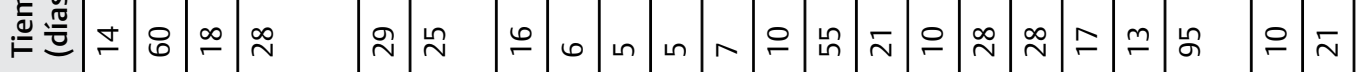

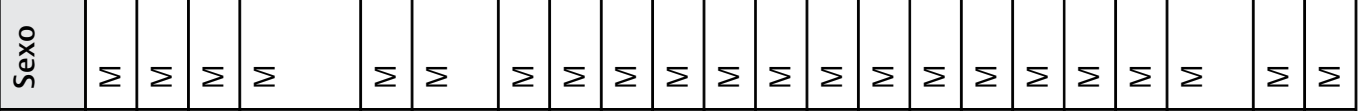

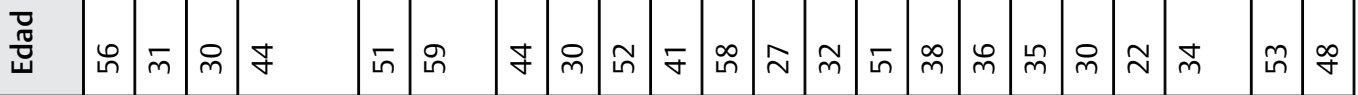

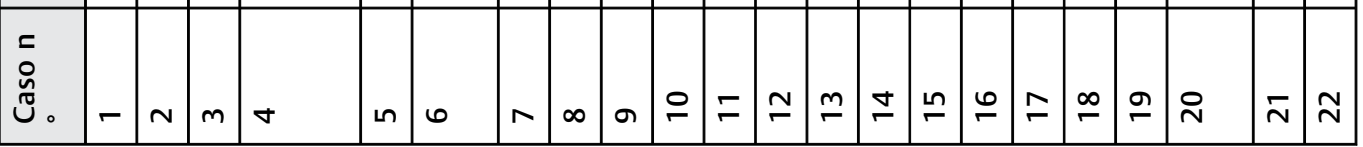

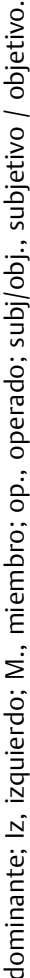



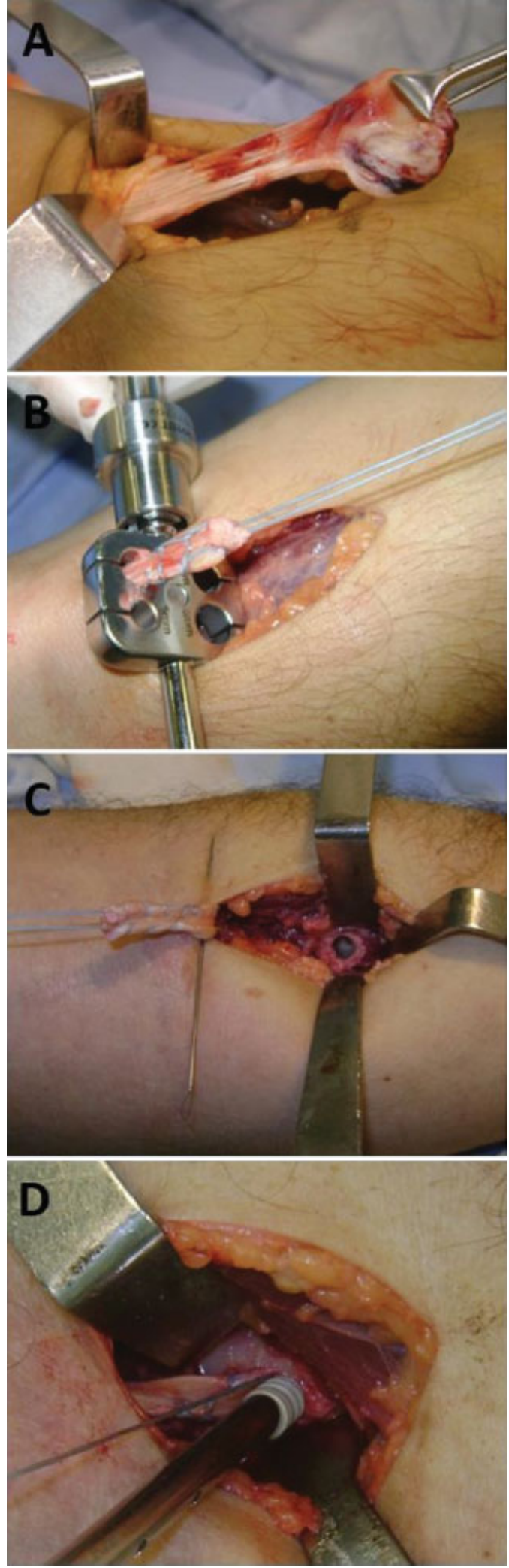

Fig. 2 A. Tendón avulsionado y recuperado por la mini-incisión anterior. B. Preparación del cabo tendinoso con punto de Krackow que pasa por el probador de $7 \mathrm{~mm}$ de diámetro. C. Túnel óseo en tuberosidad del radio. D. Tendón introducido en el túnel mediante botón cortical y fijado con tornillo interferencial.
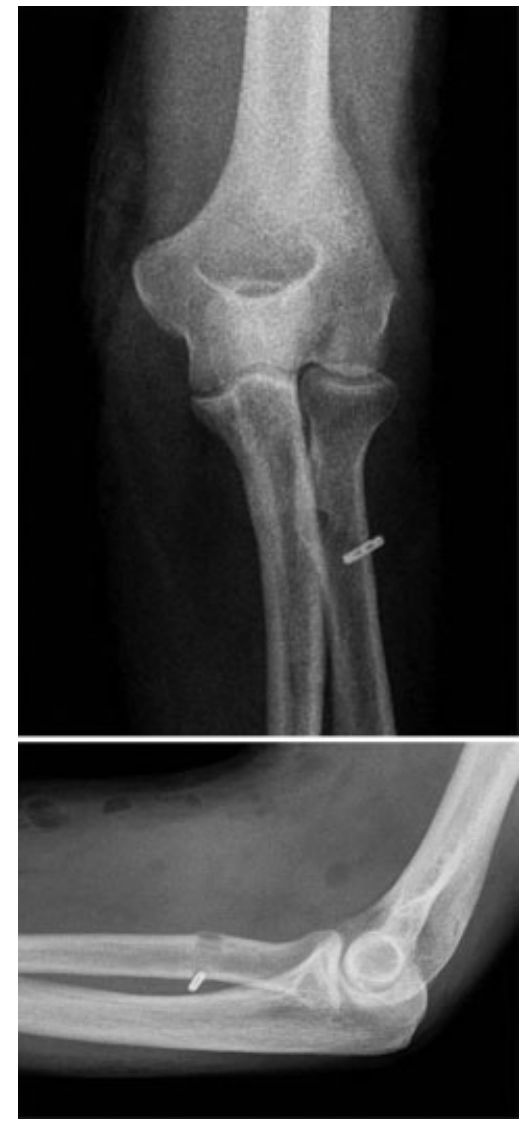

Fig. 3 Control radiológico postoperatorio anteroposterior y lateral.

del codo flexionado. La lesión se produce casi exclusivamente en hombres (93\%), en su mayoría trabajadores manuales de fuerza y deportistas, de entre 40 y 50 años, y predomina en el miembro dominante (86\%). La influencia de factores, tales como el consumo de esteroides anabólicos, ciertos medicamentos y el hábito de fumar es discutida como causa de disminución del aporte vascular y tendinosis, que pueden preceder a la rotura. ${ }^{21}$

Aunque el tratamiento conservador puede ser una opción para casos muy seleccionados, la reparación quirúrgica con reinserción anatómica a la tuberosidad, dentro de las primeras 3 ó 4 semanas, es la regla para pacientes activos. En una serie de 10 pacientes, Morrey y col. ${ }^{22}$ compararon los resultados con tratamiento conservador ( 3 pacientes), reinserción anatómica en período agudo ( 5 pacientes) y reconstrucción luego del mes ( 2 pacientes, uno con reinserción anatómica y el otro con sutura al braquial anterior).

Los pacientes sometidos al tratamiento conservador tuvieron una pérdida del $40 \%$ de la fuerza de supinación y del $30 \%$ de flexión; las reinserciones en el período agudo recuperaron, al año de la intervención, una fuerza normal de supinación y flexión; en el paciente tratado con reinserción anatómica en el período crónico, mejoró la fuerza para ambos movimientos, pero sin alcanzar la normalidad; finalmente, el caso de sutura al braquial anterior (no anatómica) mostró una pérdida de más del $50 \%$ para la supinación del antebrazo. 

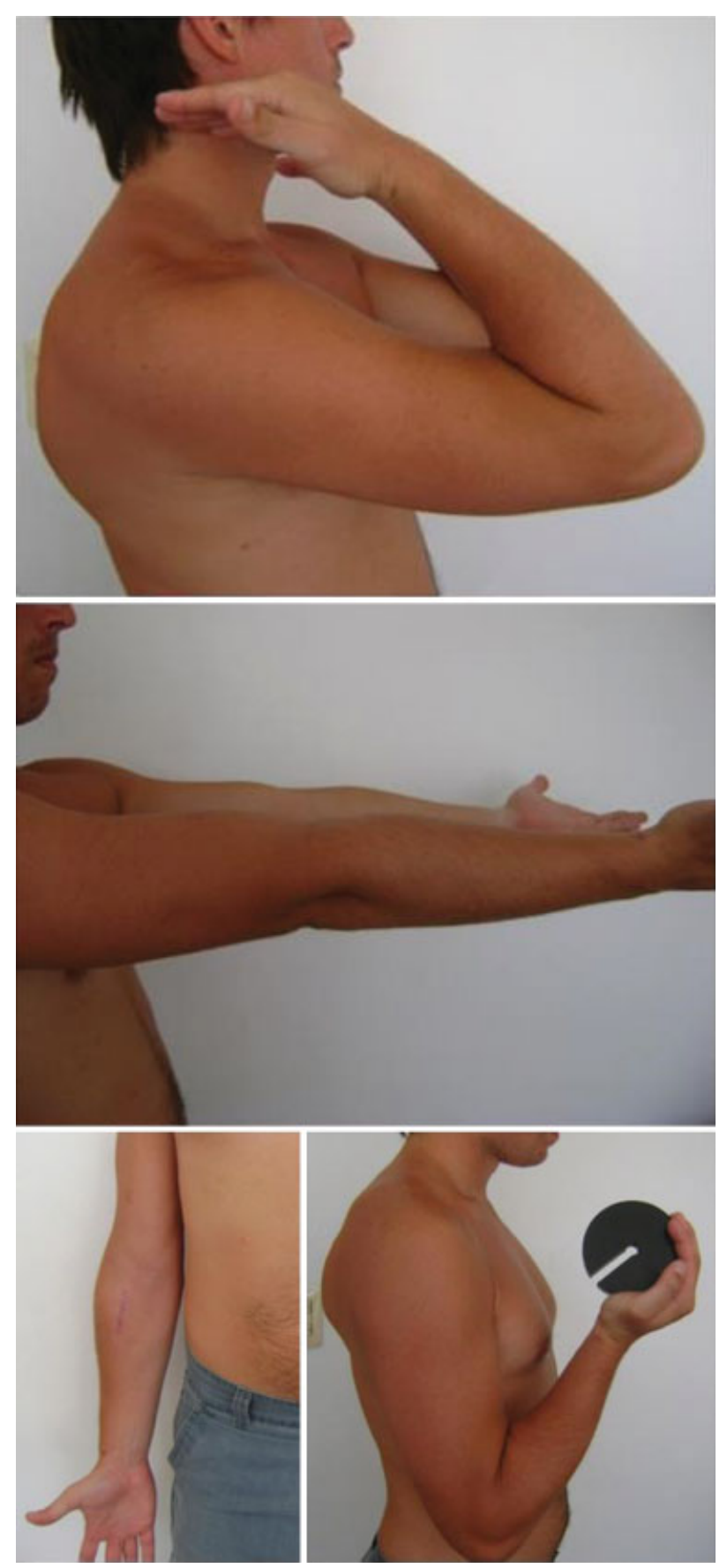

Fig. 4 Resultado clínico al mes de la cirugía, movilidad activa completa y comienzo de trabajos de carga progresiva.

Esos resultados, rubricados al mismo tiempo por otros autores $^{1,2}$, definen claramente que la reinserción del tendón a la tuberosidad bicipital en el período agudo, es superior a cualquier otro modelo de tratamiento en la búsqueda de una normal potencia de flexión y supinación.

En cuanto a los aspectos técnicos, el debate se plantea en torno a la vía de abordaje y el método de fijación. La técnica ideal sería aquella que garantice una reconstrucción anatómica y condiciones mecánicas suficientes para la aplicación de protocolos de movilización temprana controlada, con poca morbilidad y bajo índice de complicaciones.
Los abordajes han mostrado a través del tiempo una evolución tendiente a minimizar el traumatismo quirúrgico y disminuir el índice de complicaciones. Así, la vía anterior extendida de Henry ${ }^{13}$, inicialmente utilizada para la reconstrucción del bíceps distal y asociada a alta incidencia de lesiones nerviosas, es hoy sustituida por un miniabordaje transversal o longitudinal de $4 \mathrm{~cm}$, sobre la fosa antecubital, que permite exponer la tuberosidad bicipital a través del intervalo entre el braquiorradial y el pronador redondo. Las claves de ese abordaje son: a) identificar y proteger el nervio cutáneo lateral del antebrazo, b) no retraer con separadores el plano muscular lateral del abordaje para evitar el traumatismo del nervio interóseo posterior y c) proteger o ligar las venas del plexo de Henry y las ramas de la arteria recurrente radial anterior evitando la formación de hematomas y osificaciones heterotópicas. El anclaje del tendón al hueso es posible a través de ese único miniabordaje utilizando como medios de fijación arpones, botón cortical o tornillo interferencial.

La técnica de doble incisión presentada por Boyd y Anderson, en $1961,{ }^{14}$ incluye originalmente una exposición anterior amplia sobre la fosa antecubital, a través de la cual se localiza y extrae el tendón del bíceps, luego se palpa la tuberosidad del radio y una pinza curva se avanza por el espacio interóseo palpando su extremo en el dorso del antebrazo proximal. El abordaje posterior incluye la elevación de un colgajo muscular del supinador corto desde la cara lateral del cúbito, a través de la membrana interósea, hasta el radio, para exponer la tuberosidad bicipital, en máxima pronación, y proteger el nervio interóseo posterior. Esta disección subperióstica fue considerada causa de las frecuentes osificaciones heterotópicas y sinostosis radiocubitales relacionadas con esta técnica. Con el fin de disminuir esa complicación, Kelly y col. ${ }^{7}$ simplificaron ese abordaje a través de las fibras del cubital posterior y evitando disecciones extensas sobre el cúbito. Asimismo, el abordaje anterior puede simplificarse por una mini-incisión como ya fue descrito.

En una serie de 53 pacientes operados por vía anterior única $^{12}$ se comunicaron 4 complicaciones: una infección superficial, dos neuropraxias transitorias del nervio cutáneo lateral antebraquial y una parálisis del nervio interóseo posterior que se curó a las 6 semanas. El-Hawary y col. ${ }^{3}$, en un estudio prospectivo no aleatorizado, reportan un $10 \%$ de complicaciones con la doble incisión (neuropraxia transitoria del nervio radial superficial) y un $44 \%$ con la vía anterior única (la mayoría parestesias transitorias del nervio cutáneo lateral del antebrazo y una osificación heterotópica).

En nuestra serie, no hemos tenido complicaciones neurológicas ni osificación heterotópica, atribuibles al abordaje anterior único utilizado.

Muchos estudios biomecánicos se han dirigido a establecer la eficacia de los diferentes métodos de fijación y la rigidez de la construcción. Mazzocca y col. ${ }^{5}$ evaluaron, en 63 codos cadavéricos frescos-congelados, el comportamiento de 4 diferentes técnicas de anclaje (sutura transósea con técnica de Morrey, botón de fijación extracortical, tornillo de biotenodesis y fijación cortical con 2 arpones). Determinaron que el Endobutton soportó la mayor carga previa al fallo 
$(440 \mathrm{~N})$, en tanto que el tornillo interferencial ofreció la menor resistencia (232 N); sin embargo, este último fue el modelo que menor deslizamiento tendinoso permitió ante la aplicación de cargas cíclicas (2,15 mm). En ese aspecto, la fijación con sutura transósea fue la menos eficaz $(3,55 \mathrm{~mm})$ seguida del Endobutton $(3,42 \mathrm{~mm})$ y la construcción con arpones (2,33 mm). Esos hallazgos demuestran que, con esas últimas técnicas, la movilización temprana en el postoperatorio produce el pistoneo del tendón dentro del túnel, retarda o inhibe la osteointegración y predispone al fallo. Todas las formas de reconstrucción mostraron una resistencia superior a la carga necesaria para provocar la rotura de un tendón distal de bíceps intacto (204,3 N, en promedio), según estudios de Idler y col. ${ }^{23}$

Sobre la base de esos estudios biomecánicos, ese grupo introduce un nuevo concepto en la fijación del bíceps distal combinando el uso del botón cortical, con una modificación de la original técnica de Bain ${ }^{10}$ (Tension-Slide Technique) y el tornillo interferencial de biotenodesis. Esa doble fijación utiliza el implante más resistente a la carga antes del fallo (Endobutton) y el que menor desplazamiento tendinoso mostró ante la aplicación de cargas cíclicas (tornillo de biotenodesis). Así, se aplican los principios anatómicos, biológicos y biomecánicos más favorables para una rápida y fisiológica integración osteotendinosa.

Las complicaciones comunicadas más frecuentes ${ }^{24}$ son las lesiones nerviosas (neuritis del nervio cutáneo lateral antebraquial -nervio interóseo posterior- nervio radial sensitivo) y osificación heterotópica, muchas de ellas relacionadas con las dificultades que plantea una reconstrucción después de las 4 semanas. En ese sentido, Kelly y col. ${ }^{7}$ informan un 31\% de complicaciones con la técnica de doble incisión y sutura transósea, y destacan que ellas aumentan en relación directa con el tiempo de evolución.

En nuestro grupo de pacientes, el índice de complicaciones fue menor que en otras series, una fístula aséptica sobre la incisión adicional en cara anterior del brazo para liberar adherencias en un paciente que llevaba más de un mes del traumatismo, se curó luego de una limpieza quirurgica y vendaje compresivo (Caso 4). Las neuropraxias altas del nervio radial fueron consecuencia del manguito hemostático y se curaron por completo a los 50 días (Casos 6 y 20). Ninguna de esas complicaciones alteró el protocolo de rehabilitación ni los resultados funcionales obtenidos.

En una serie de 19 pacientes publicada recientemente ${ }^{25}$, se demuestra un efecto de osteólisis progresiva en el radio utilizando el tornillo interferencial de biotenodesis como único medio de fijación. Con un seguimiento promedio de 22 meses, los autores constataron un aumento de la relación: diámetro del túnel-sección transversa del radio, entre el posoperatorio inmediato (49\%) y el control final (61\%). Solo 8 de los 19 pacientes tuvieron manifestaciones clínicas. En nuestro estudio, no hemos observado esa complicación en los estudios radiológicos posoperatorios alejados.

La ausencia de osificaciones heterotópicas en nuestro grupo puede atribuirse al abordaje anatómico y poco traumático, a la irrigación constante durante el fresado y a la movilización temprana que permite la doble fijación.
Por último, al comparar, con el mismo puntaje de evaluación, nuestros resultados con los de otra serie que utiliza la técnica de doble fijación, ${ }^{26}$ observamos que son muy similares e igualmente satisfactorios (puntajes promedio de Andrews y Carson: 193,2 puntos y 196 puntos, respectivamente). Asimismo, las complicaciones ocurrieron con una frecuencia que no arrojó diferencias estadísticamente significativas, promedio $13,6 \%$ y $9,8 \%$, respectivamente.

Si bien debe aceptarse que el uso de 2 implantes para efectuar la tenodesis encarece el costo del procedimiento, los buenos resultados, el bajo porcentaje de complicaciones y el retorno más rápido a las actividades de la vida diaria, laborales y deportivas, la convierten en nuestra primera opción terapéutica en pacientes jóvenes, activos y de alta demanda funcional.

Las debilidades de este estudio se relacionan con su diseño observacional descriptivo, el moderado número de casos y el tiempo de seguimiento. Asimismo, la ausencia de grupo control y de comparación de resultados con otros procedimientos no permite considerar a la doble fijación como superior a las demás. Futuros estudios prospectivos, comparativos y aleatorizados definirán, quizás, qué técnica quirurgica es la más eficaz para esta patología.

\section{Conclusión}

La técnica basada en incisión anterior única y doble fijación para el tendón distal del bíceps ha demostrado ser segura y eficaz, brinda resultados satisfactorios y la tasa de complicaciones es baja. Los protocolos de movilización temprana hacen posible un retorno más rápido a las actividades de la vida diaria, laborales y deportivas.

\section{Conflictos de interese}

Los autores no declaran ningún conflictos de interese.

\section{Bibliografía}

1 Baker BE, Bierwagen D. Rupture of the distal tendon of the biceps brachii. Operative versus non-operative treatment. J Bone Joint Surg Am 1985;67(03):414-417

2 Hetsroni I, Pilz-Burstein R, Nyska M, Back Z, Barchilon V, Mann G. Avulsion of the distal biceps brachii tendon in middle-aged population: is surgical repair advisable? A comparative study of 22 patients treated with either nonoperative management or early anatomical repair. Injury 2008;39(07):753-760

3 El-Hawary R, Macdermid JC, Faber KJ, Patterson SD, King GJ. Distal biceps tendon repair: comparison of surgical techniques. J Hand Surg Am 2003;28(03):496-502

4 Mazzocca AD, Bicos J, Arciero RA, Romeo AA, Cohen MS, Nicholson G. Repair of distal biceps tendon ruptures using a combined anatomic interference screw and cortical button. Tech Shoulder Elbow Surg 2005;6(02):108-115

5 Mazzocca AD, Burton KJ, Romeo AA, Santangelo S, Adams DA, Arciero RA. Biomechanical evaluation of 4 techniques of distal biceps brachii tendon repair. Am J Sports Med 2007;35(02):252-258

6 Sethi P, Cunningham J, Miller S, Sutton K, Mazzocca A. Anatomical repair of the distal biceps tendon using the Tension-Slide Technique. Tech Shoulder Elbow Surg 2008;9(04):182-187

7 Kelly EW, Morrey BF, O'Driscoll SW. Complications of repair of the distal biceps tendon with the modified two-incision technique. J Bone Joint Surg Am 2000;82-A(11):1575-1581 
8 John CK, Field LD, Weiss KS, Savoie FH III. Single-incision repair of acute distal biceps ruptures by use of suture anchors. J Shoulder Elbow Surg 2007;16(01):78-83

9 Kobayashi K, Bruno RJ, Cassidy C. Single anterior incision suture anchor technique for distal biceps tendon ruptures. Orthopedics 2003;26(08):767-770

10 Bain GI, Prem H, Heptinstall RJ, Verhellen R, Paix D. Repair of distal biceps tendon rupture: a new technique using the Endobutton. J Shoulder Elbow Surg 2000;9(02):120-126

11 Silva J, Eskander MS, Lareau C, DeAngelis NA. Treatment of distal biceps tendon ruptures using a single-incision technique and a Bio-Tenodesis screw. Orthopedics 2010;33(07):477

12 McKee MD, Hirji R, Schemitsch EH, Wild LM, Waddell JP. Patientoriented functional outcome after repair of distal biceps tendon ruptures using a single-incision technique. J Shoulder Elbow Surg 2005; 14(03):302-306

13 Fischer WR, Shepanek LA. Avulsion of the insertion of the biceps brachii; report of a case. J Bone Joint Surg Am 1956;38-A(01): 158-159

14 Boyd HB, Anderson LD. A method for reinsertion of the distal biceps brachii tendon. J Bone Joint Surg Am 1961;43:1041-1043

15 Phadnis J, Bain G. Endoscopic-assisted Distal Biceps Footprint Repair. Tech Hand Up Extrem Surg 2015;19(02):55-59

16 Capomassi MA, Gardenal RM, Slullitel M, Suárez E, Benedetto I. Rotura del bíceps distal: Evaluación de resultados con técnica de doble fijación. Rev Asoc Argent Ortop Traumatol 2013;78(04):171-179

17 Hudak PL, Amadio PC, Bombardier C; The Upper Extremity Collaborative Group (UECG). Development of an upper extremity outcome measure: the DASH (disabilities of the arm, shoulder and hand) [corrected]. Am J Ind Med 1996;29(06):602-608

18 Andrews JR, Carson WG. Arthroscopy of the elbow. Arthroscopy 1985;1(02):97-107

19 Medical Research Council. Nerve Injuries Committee. Results of nerve suture. En: Seddon $\mathrm{H}$, ed. Peripheral Nerve Injuries. London: Her Majesty's Stationery Office; 1954

20 Mackinnon SE, Dellon AL. Results of nerve repair and grafting: Surgery of the Peripheral Nerve. New York: Thieme; 1988, p. 115

21 Sutton KM, Dodds SD, Ahmad CS, Sethi PM. Surgical treatment of distal biceps rupture. J Am Acad Orthop Surg 2010;18(03):139-148

22 Morrey BF, Askew LJ, An KN, Dobyns JH. Rupture of the distal tendon of the biceps brachii. A biomechanical study. J Bone Joint Surg Am 1985;67(03):418-421

23 Idler CS, Montgomery WH III, Lindsey DP, Badua PA, Wynne GF, Yerby SA. Distal biceps tendon repair: a biomechanical comparison of intact tendon and 2 repair techniques. Am J Sports Med 2006;34(06):968-974

24 Cohen MS. Complications of distal biceps tendon repairs. Sports Med Arthrosc Rev 2008;16(03):148-153

25 Potapov A, Laflamme YG, Gagnon S, Canet F, Rouleau DM. Progressive osteolysis of the radius after distal biceps tendon repair with the bioabsorbable screw. J Shoulder Elbow Surg 2011; 20(05):819-826

26 Heinzelmann AD, Savoie FH III, Ramsey JR, Field LD, Mazzocca AD. A combined technique for distal biceps repair using a soft tissue button and biotenodesis interference screw. Am J Sports Med 2009;37(05):989-994 